\title{
Growth modes of thin films of ligand-free metal clusters
}

\author{
A. Dollinger, ${ }^{1}$ C. H. Strobel, ${ }^{1}$ H. Bleuel, ${ }^{1}$ A. Marsteller, ${ }^{1}$ G. Gantefoer, ${ }^{1, a)}$ D. H. Fairbrother, ${ }^{2}$ \\ Xin Tang, ${ }^{2}$ K. H. Bowen, ${ }^{2}$ and Young Dok Kim ${ }^{3}$ \\ ${ }^{1}$ Department of Physics, University of Konstanz, Konstanz, Germany \\ ${ }^{2}$ Departments of Chemistry and Materials Science, Johns Hopkins University, Baltimore, Maryland MD 21218 , \\ USA \\ ${ }^{3}$ Department of Chemistry, Sungkyunkwan University, 440-746 Suwon, South Korea
}

\begin{abstract}
Size-selected $\mathrm{Mo}_{\mathrm{n}}{ }^{-}, \mathrm{W}_{\mathrm{n}}{ }^{-}$, and $\mathrm{Fe}_{\mathrm{n}}{ }^{-}$cluster anions are deposited on a weakly interacting substrate (highly oriented pyrolytic graphite) and studied ex-situ using atomic force microscopy. Depending on size, three growth modes can be distinguished. Very small clusters consisting of less than 10-30 atoms behave similar to atoms and coalesce into 3-dimensional bulk-like islands. Medium sized clusters consisting of hundreds of atoms do not coalesce and follow a Stanski-Krastanov growth pattern. At low coverage, an almost perfect monolayer is formed. This is a new finding different from all previous studies on deposited metal clusters. For clusters with several thousands of atoms, the growth pattern again changes. At low coverage, the substrate is dotted with individual clusters, while at high coverage, the surface becomes extremely rough.
\end{abstract}

\section{INTRODUCTION}

Clusters contain an ensemble of bound atoms whose size is intermediate between an individual atom and a bulk solid. As such, clusters are a unique class of materials, which continue to attract intense scientific interest due to the fact that they often exhibit extraordinary electronic, optical, and catalytic properties, etc. ${ }^{1-3}$ Metal clusters are of particular interest. Many of their features are strongly sizedependent, e.g., gold clusters catalyze $\mathrm{CO}$ oxidization at low temperatures, whereas bulk gold is chemically inert. ${ }^{4}$ Synthesizing materials from highly functional metal clusters may open the gateway to the creation of novel classes of materials. Such materials can be used in a multitude of applications like catalysts, electrode materials, sensors, or solar cells, etc., and therefore efforts have been undertaken to synthesize them. ${ }^{5}$ However, for metal clusters, there is a major obstacle. A sound synthesis route to materials made from metal clusters has to overcome the problem of coalescence resulting in an immediate loss of their original features. One way might be the use of ligand-stabilized metal clusters and in fact these condense into highly ordered crystals without the problem of coalescence. ${ }^{6}$ Nevertheless, ligands isolate clusters and in consequence change their physical and chemical properties. In order to keep the attributes of bare clusters, an alternative and more promising method is size-selected cluster formation in the gas phase and deposition under soft landing conditions. ${ }^{7,8}$ So far this technique has been successfully used to investigate the properties of individual deposited clusters. ${ }^{9-19}$ In these studies, coalescence is circumvented by dramatically lowering the coverage and/or immobilizing clusters on the surface. Attempts to synthesize cluster thin films by increasing the coverage have failed since deposited clusters merged into larger particles. ${ }^{20-22}$ Depending on the preparation

${ }^{\text {a)} E-m a i l: ~ g e r d . g a n t e f o e r @ u n i-k o n s t a n z . d e ~}$ conditions, 3-dimensional islands of various sizes and shapes are formed. Similar islands can also be prepared by the deposition of atoms. ${ }^{23-25}$ Therefore, the formation of complex island shapes is not an effect unique to clusters. The clusters coalesce and lose all their nano-properties.

The coalescence of most metal clusters is a consequence of the lower melting points of very small particles. ${ }^{26,27}$ Especially, the low coordinated surface atoms are more or less liquid at room temperature and two clusters touching each other merge into a larger particle similar to two drops of water. This holds for clusters of materials with relatively low melting points such as $\mathrm{Ag}, \mathrm{Au}, \mathrm{Bi}$, and $\mathrm{Sb}$. Recently, we found that coalescence at room temperature can be avoided for clusters of high melting point materials such as molybdenum and tungsten. ${ }^{28}$ Scanning tunnelling microscope (STM) pictures revealed that the cluster thin films consist of individual clusters.

So far, our efforts focussed on the proof that the films indeed consist of individual clusters. Here, we systematically study the growth modes of these films on a weakly interacting substrate, namely, highly oriented pyrolytic graphite (HOPG). Metal atoms are highly mobile on such a substrate and coalesce into islands. Since the bond between the metal atoms is much stronger than the interaction with the substrate, only VolmerWeber growth is observed. The atoms form 3-dimensional islands. The medium sized clusters studied here show a different behavior. At low coverage, they form an almost perfect monolayer indicating a Stranski-Krastanov growth mode. Very large clusters with more than 1000 atoms have a low mobility and stick to the landing site. At low coverage, the substrate is dotted with individual clusters and at higher coverage the surface is extremely rugged. Hence, thin film growth of noncoalescing clusters strongly depends on size and at least three different regimes can be distinguished. Especially, the formation of a perfect monolayer is a unique nanoeffect and a novelty for metals on Van-der-Waals surfaces. 


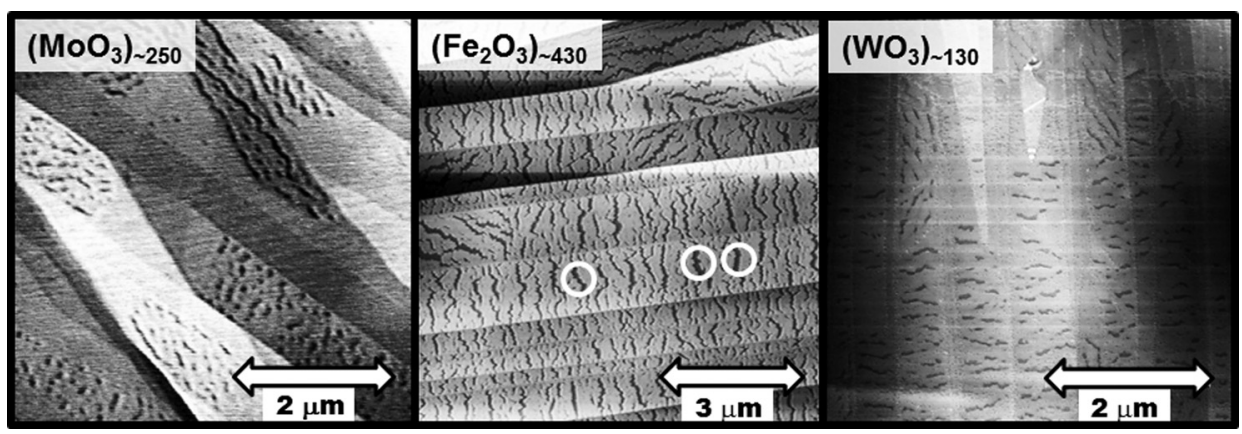

FIG. 1. Ex-situ AFM pictures of cluster thin films on HOPG. The coverage is slightly below the completion of a cluster monolayer. Most of the surface is covered by a smooth cluster monolayer. Left: $\left(\mathrm{MoO}_{3}\right)_{250 \pm 13}$; middle: $\left(\mathrm{Fe}_{2} \mathrm{O}_{3}\right)_{430 \pm 22}$; right: $\left(\mathrm{WO}_{3}\right)_{130 \pm 7}$. For the cracks marked by the white circles, height profiles have been recorded (Fig. 2).

\section{EXPERIMENTAL}

The experimental set-up has been described in detail elsewhere. ${ }^{22,28-31}$ In short, $\mathrm{Mo}_{\mathrm{n}}{ }^{-}, \mathrm{W}_{\mathrm{n}}{ }^{-}$, and $\mathrm{Fe}_{\mathrm{n}}{ }^{-}$cluster anions are produced via magnetron sputtering, mass-selected with a sector magnet (mass-resolution $\mathrm{m} / \Delta \mathrm{m}=20$ ), and deposited within the soft-landing criterion at high-vacuum conditions ( $\mathrm{p}<1 \times 10^{-8} \mathrm{mbar}$ ) on HOPG. Due to the limited mass resolution in all experiments, a range of slightly different masses is deposited. The width of the mass distribution is $\pm 5 \%$ around the nominal mass. After deposition, the topography of the surfaces is investigated by $e x$-situ atomic force microscopy (AFM). The AFM is operated in the intermittent contact mode (tapping mode). To demonstrate the cleanliness of the grown material, samples are characterized by ex-situ XPS. All experiments are conducted at room temperature.

\section{RESULTS}

There are three size regimes: small clusters with less than $10-30$ atoms, ${ }^{28}$ medium sized clusters with up to 1000 atoms and larger clusters consisting of more than 1000 atoms. The present work focusses on medium-sized and large clusters. In detail, the following cluster sizes have been deposited (size range $\pm 5 \%$ ):

$$
\begin{aligned}
\text { Mo }: \mathrm{n} & =50,250,274,1250,3350, \\
\mathrm{~W}: \mathrm{n} & =27,130,660,1300,3500, \\
\text { Fe }: \mathrm{n} & =30,430,1200 .
\end{aligned}
$$

On each sample, in the centre of the deposition spot, the film thickness is of the order of several $100 \mathrm{~nm}$. With increasing distance to the centre, the coverage decreases down to submonolayer coverage. Accordingly, on a single sample, it is possible to study the coverage dependence of the thin film growth mode by analysing different areas.

For all samples, ex-situ AFM pictures have been recorded. XPS spectra show, that in most cases, the clusters are completely oxidized. For large Mo and W clusters, complete oxidation is reached only after several hours of exposure to ambient conditions. For these clusters, short exposure times (15 min) result in surface oxidation only. The thin films studied here are completely oxidized. Fig. 1 displays AFM pictures of medium sized $\left(\mathrm{MoO}_{3}\right)_{\mathrm{n}}$, $\left(\mathrm{WO}_{3}\right)_{\mathrm{n}}$, and $\left(\mathrm{Fe}_{2} \mathrm{O}_{2}\right)_{\mathrm{n}}$ clusters on HOPG. The coverage corresponds roughly to a cluster monolayer. All three AFM pictures show a smooth surface with step edges corresponding to the step edges of the underlying HOPG substrate. Even without further analysis, these pictures indicate the formation of a monolayer corresponding to a Stransky-Krastanov growth mode. In all three cases, the monolayer is not completely closed but exhibits cracks. Height profiles across these cracks show that the depth corresponds roughly to the diameter of the clusters (Fig. 2). The surfaces of the cluster layers are rather smooth similar to the HOPG surface.

At lower coverage, 2-dimensional islands are formed (Fig. 3, left). For these islands, the heights have been studied systematically using the AFM. For each sample, ten roughly circular islands with a diameter of $\sim 10 \mathrm{~nm}$ have been selected. For each island, several height profiles in different directions have been recorded from which the height is determined. On one sample, all scans for all such islands produce similar heights. Table I shows a comparison of the measured island heights with the calculated diameters of the oxidized clusters. There is a limited but recognizable agreement. The islands heights of some samples are significantly lower than the respective calculated cluster diameters $\left(\left(\mathrm{WO}_{3}\right)_{3500}\right.$ and $\left.\left(\mathrm{Fe}_{2} \mathrm{O}_{3}\right)_{1200}\right)$. It is worth noting that the cluster height was calculated based on the assumption of a spherical cluster shape, whereas the structures of clusters on a surface may be distorted by the substrate-cluster interactions.

In general, the measured island thickness increases with increasing cluster size. There is one exception. For $\left(\mathrm{Fe}_{2} \mathrm{O}_{3}\right)_{30}$, the measured island thickness is larger than the one for $\left(\mathrm{Fe}_{2} \mathrm{O}_{3}\right)_{430}$. Fig. 4 displays the island height distributions measured for $\left(\mathrm{Fe}_{2} \mathrm{O}_{3}\right)_{30}$ and for $\left(\mathrm{Fe}_{2} \mathrm{O}_{3}\right)_{1200}$ clusters. For the large clusters, there is only a small scatter around the mean value, but for the small clusters a relatively broad distribution is found. This is regarded as a sign for coalescence. In the previous studies, it was suggested that such cluster agglomeration can only be observed for clusters consisting of $\sim 20$ atoms or less in the case of $\mathrm{Mo} .{ }^{28} \mathrm{Fe}$ has a considerably lower

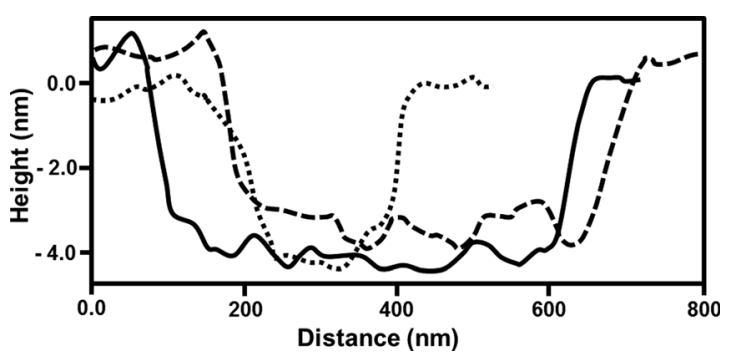

FIG. 2. Height profiles recorded for the three cracks marked in the AFM picture of the $\left(\mathrm{Fe}_{2} \mathrm{O}_{3}\right)_{430 \pm 22}$ sample in Fig. 1. All cracks have the same depth which corresponds roughly to the diameter of the deposited clusters. The top of the cluster layer is as smooth as the surface of the HOPG. 


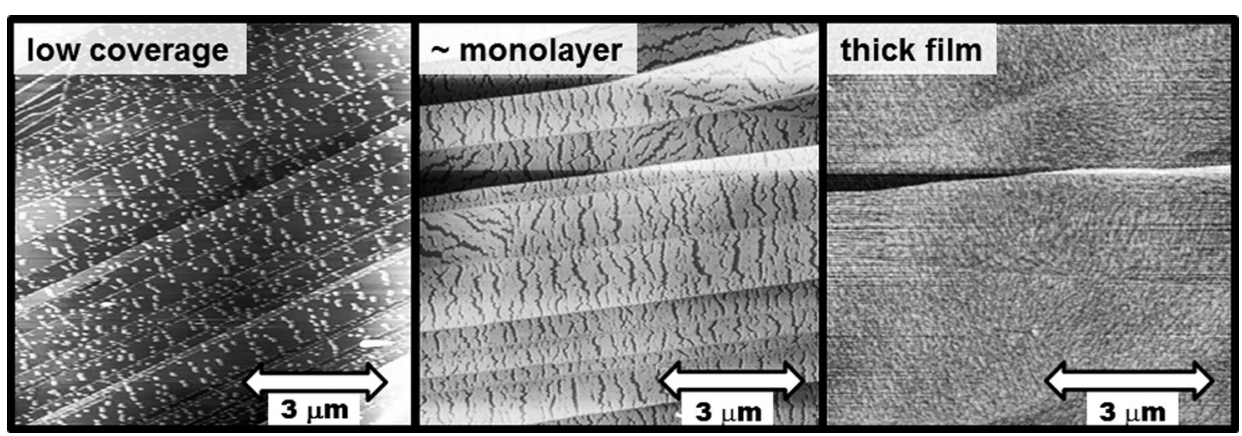

FIG. 3. Ex-situ AFM images of $\left(\mathrm{Fe}_{2} \mathrm{O}_{3}\right)_{430 \pm 22}$ clusters deposited on HOPG at different cluster coverages.

TABLE I. Comparison of the measured heights of the islands on HOPG with the calculated diameters of the oxidized clusters.

\begin{tabular}{lcc}
\hline \hline Cluster & Calculated diameter & Measured island height \\
\hline$\left(\mathrm{MoO}_{3}\right)_{50 \pm 3}$ & $1.69 \pm 0.05$ & $1.78 \pm 0.27$ \\
$\left(\mathrm{MoO}_{3}\right)_{250 \pm 13}$ & $1.90 \pm 0.10$ & $2.11 \pm 0.16$ \\
$\left(\mathrm{MoO}_{3}\right)_{1300 \pm 65}$ & $5.02 \pm 0.17$ & $5.54 \pm 0.25$ \\
$\left(\mathrm{WO}_{3}\right)_{27 \pm 2}$ & $1.40 \pm 0.04$ & $1.25 \pm 0.14$ \\
$\left(\mathrm{WO}_{3}\right)_{130 \pm 7}$ & $2.37 \pm 0.08$ & $2.12 \pm 0.31$ \\
$\left(\mathrm{WO}_{3}\right)_{3500 \pm 180}$ & $7.11 \pm 0.25$ & $5.42 \pm 0.61$ \\
$\left(\mathrm{Fe}_{2} \mathrm{O}_{3}\right)_{30 \pm 2}$ & $1.43 \pm 0.04$ & $1.70 \pm 0.40$ \\
$\left(\mathrm{Fe}_{2} \mathrm{O}_{3}\right)_{430 \pm 22}$ & $3.63 \pm 0.12$ & $1.66 \pm 0.16$ \\
$\left(\mathrm{Fe}_{2} \mathrm{O}_{3}\right)_{1200 \pm 60}$ & $4.87 \pm 0.19$ & $2.64 \pm 0.37$ \\
\hline
\end{tabular}
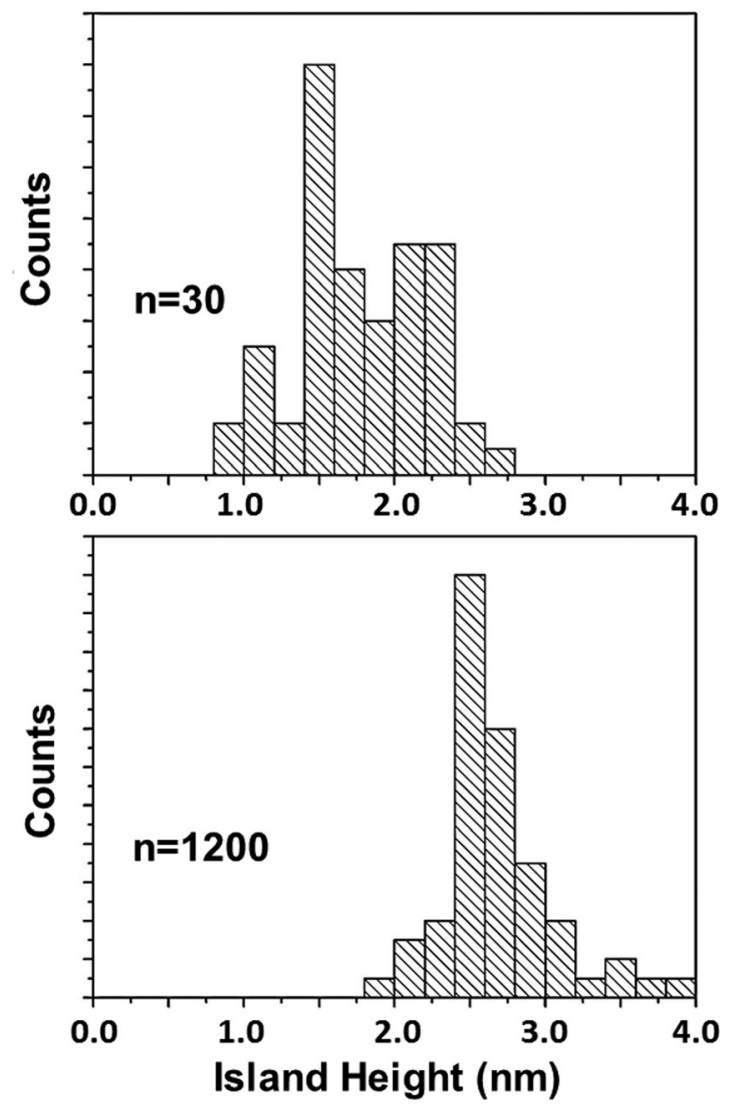

FIG. 4. Distribution of island heights for $\left(\mathrm{Fe}_{2} \mathrm{O}_{3}\right)_{30 \pm 2}$ and for $\left(\mathrm{Fe}_{2} \mathrm{O}_{3}\right)_{1200 \pm 60}$ clusters measured with the AFM. melting point than Mo and $\mathrm{W}$, and it is quite possible that the Fe clusters with 30 atoms coalesce, whereas similarly sized W clusters do not. Apart from this exception, the heights correspond roughly to the calculated diameters of the clusters.

At coverages above a cluster monolayer, the film growth follows the Stanski-Krastanov pattern. The surfaces become rough (Fig. 3, right) and it is not possible to resolve any internal structure with the AFM except for the biggest clusters (see below). A few samples have been studied ex-situ with a STM. Fig. 5 shows an example of a thin film consisting of $\left(\mathrm{Fe}_{2} \mathrm{O}_{3}\right)_{430}$ clusters. The rough surface supports the notion of a Stranski-Krastanov growth.

For larger clusters, the pattern changes again. The upper part of Fig. 6 displays an AFM picture of a HOPG surface covered with $\left(\mathrm{MoO}_{3}\right)_{3500 \pm 180}$ clusters at low coverage. No islands are observed. Instead, the clusters seem to remain where they landed indicating a low mobility. However, the mobility is not exactly zero, because the step edges are decorated with clusters. For these large clusters, the AFM is able to resolve the granular structure of the film surface at higher coverage (Fig. 6, bottom).

\section{DISCUSSION}

A metal bond is relatively strong while the Van-derWaals interaction is weak. Accordingly, metal atoms deposited on a Van-der-Waals surface immediately merge into larger 3-dimensional islands. At room temperature, the highly mobile atoms diffuse until they are trapped at a step edge or a defect or until they collide with another metal atom. Two metal atoms form a dimer with a lower mobility.

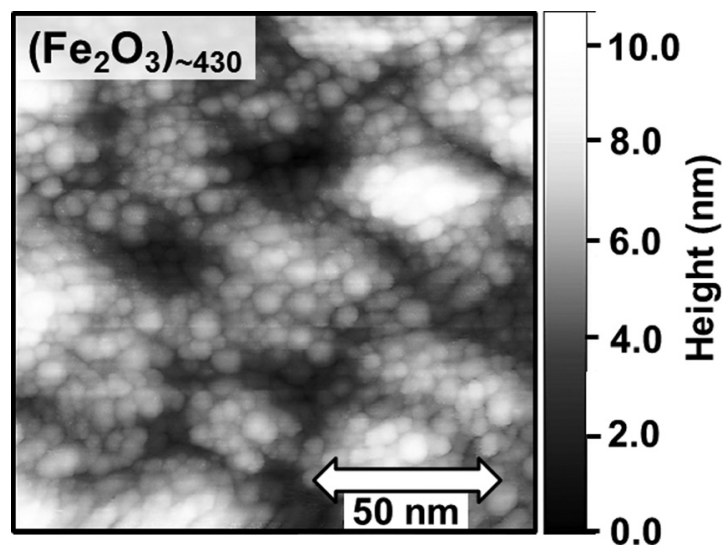

FIG. 5. Ex-situ STM picture of a cluster thin film made from $\left(\mathrm{Fe}_{2} \mathrm{O}_{3}\right)_{430 \pm 22}$ clusters. The picture has been recorded at the center of the deposition spot, where the film is more than $100 \mathrm{~nm}$ thick. 


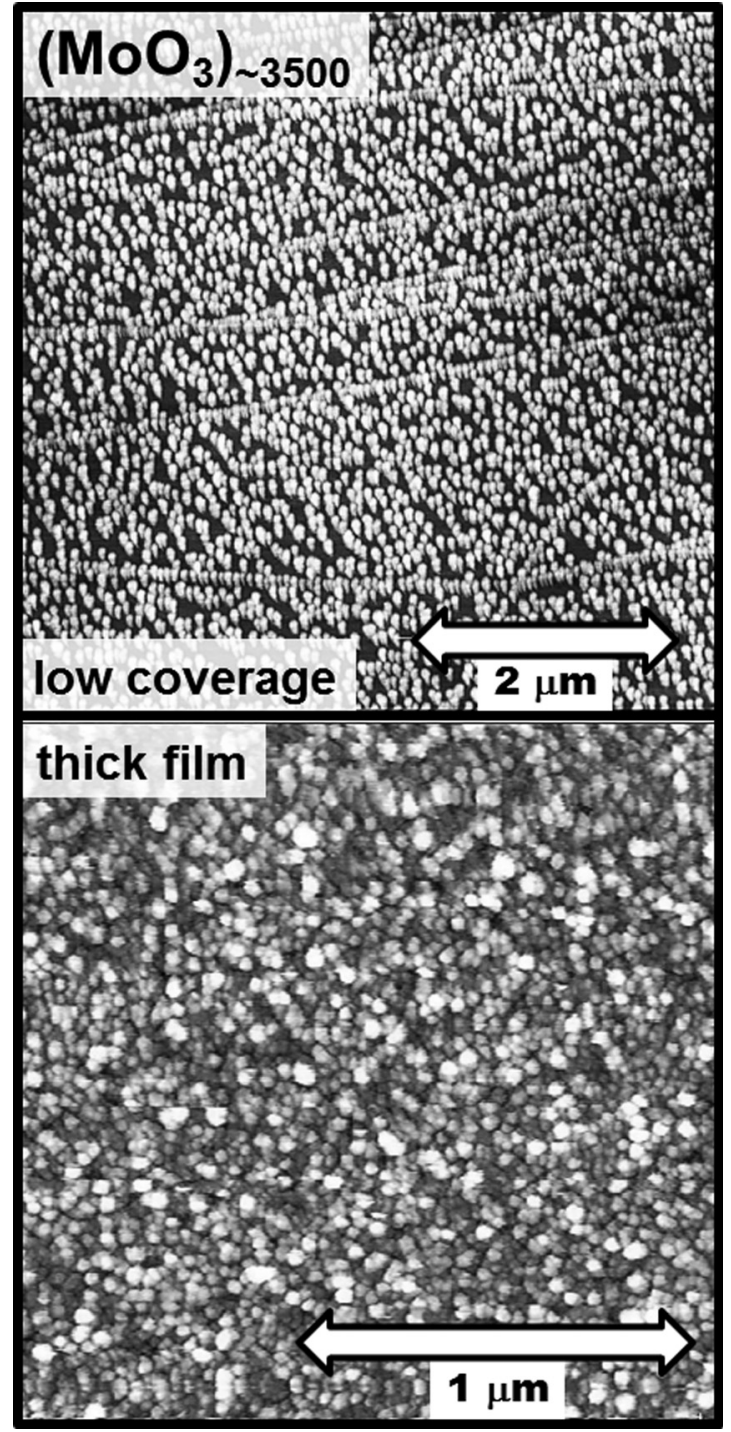

FIG. 6. Ex-situ AFM pictures of $\left(\mathrm{MoO}_{3}\right)_{3500 \pm 180}$ clusters on HOPG at low and at high coverages.

It might act as a condensation nucleus collecting more atoms. The first very few atoms form a 2-dimensional island, but this becomes unstable. In 2-dimensional islands, the average coordination number $(\sim 6)$ is far below the optimum of the bulk (12). In the limit of a vanishing interaction with the substrate, the growth pattern is similar to the condensation process in the gas phase: the atoms coalesce into 3-dimensional particles. This is Volmer-Weber growth.

Very small clusters deposited on HOPG behave probably similar to atoms. At least for dimers and trimers, there should be no barrier against coalescence. Above a certain size, the clusters do no longer coalesce on the time scale of the experiment. For merging two smaller particles into a larger one, virtually all atom positions have to be changed. For particles of high melting point materials with rigid bonds, there is barrier against such a structural rearrangement. The size limit of coalescence depends on the material and on the temperature. For high melting point metals, such as Molybdenum, the limit is about $\mathrm{n}=20$ at room temperature. ${ }^{28}$ On HOPG, such clusters with more than 20 atoms form islands consisting of individual clusters. The thin film growth mode of these clusters on HOPG should not be different from the one of atoms as long as the interaction with the Van-der-Waals substrate is weaker than the cluster-cluster interaction. These clusters are expected to follow the Vollmer-Weber growth pattern.

The experimental results contradict this expectation. At low coverage, the medium sized clusters do not form 3dimensional islands. The $e x$-situ AFM data prove that instead a 2-dimensional monolayer is formed. This could be an artefact of the experimental method. There are two possibilities. It could be that the AFM tip itself somehow modifies the cluster film. However, it is rather unlikely that the tip created the monolayer. This would only be possible if the clusters are easily moved by the tip. However, what usually happens in such a case is that the tip "cleans" the surface like a broom and moves the material at the edges of the picture. This is not observed. The other effect which might alter the morphology of the cluster film is oxidation, which takes place in the moment the sample is removed from the deposition chamber and exposed to air. Again, this effect could not be responsible for the formation of a monolayer. Most likely, the monolayer has formed prior to oxidation and survived upon oxidation under air.

According to the above considerations, the bare metal clusters follow a Stranski-Krastanov growth pattern. In the beginning of the growth process, a monolayer is formed indicating a relatively strong interaction with the substrate. A reason for such an interaction could be a charge transfer between the particles and the substrate. The work function of the HOPG is different from the one of the cluster. This results in a charge transfer and an electrostatic interaction which could be much stronger than a Van-der-Waals bond and stronger than the cluster-cluster interaction.

It is questionable why the medium sized clusters on top of the completed monolayer do not form a second homogeneous layer, but prefer a 3-dimensional growth mode. In the first layer, the cluster-substrate interaction is stronger that the cluster-cluster interaction. For the second layer, the cluster-cluster interaction could become dominant. In addition, the mobilities of the particles on the bare substrate and on the closed monolayer are different. This shift of equilibrium between the forces acting on the particles might drive the transition to a $3 \mathrm{D}$ growth mode.

Larger clusters cannot form a monolayer because of their low mobility. At zero mobility the clusters would stay at their landing sites. This leads to a Volmer-Weber growth similar to the growth pattern of the atoms. Although the general patterns are similar, the microscopic mechanisms are different. Especially at low coverage the appearances are completely different. The large clusters cover the surface uniformly (Fig. 6, top). In contrast, in the case of atoms and small clusters, most of the surface is clean and the deposited material is packed in few 3-dimensional islands.

\section{CONCLUSION}

Thin metal films are prepared by deposition of sizeselected bare metal clusters on highly oriented graphite. The morphology of the films varies with cluster size. In contrast to the earlier studies, clusters of high melting point metals 
were used. The particles do not coalesce at room temperature but form thin films of weakly bond clusters. For the growth modes, three different sizes regimes can be distinguished. Very small clusters with less than 10-30 atoms behave similar to atoms and form 3-dimensional islands (Volmer-Weber growth). The medium sized clusters with up to $\sim 1000$ atoms form a surprisingly perfect monolayer. At higher coverage, the surface becomes rugged indicating a Stranski-Krastanov growth. For even larger clusters with more than 1000 atoms, the appearance changes again. These clusters have a low mobility and stay close to their landing site. At submonolayer coverage, the sample is dotted with individual clusters. At higher coverage, the surface becomes extremely rough. This pronounced size dependence of the growth pattern of cluster thin films is a new finding again emphasising the special behavior of nanomatter. These new growth modes could be exploited for the fabrication of functionalized surfaces with extraordinary properties. For example, the formation of a cluster monolayer can be used to coat a large variety of surfaces with a very smooth metal thin film. The thickness can be controlled by the cluster size. Clusters could be used when standard coating techniques fail, because the particles adhere even to Van-der-Waals surfaces.

\section{ACKNOWLEDGMENTS}

Support by German Science Foundation (Grant No. GA $389 / 15-1$ ) is gratefully acknowledged.

A portion of this material is based on work supported by the U.S. National Science Foundation under Grant No. CHE1360692 (K.H.B.).

${ }^{1}$ A. W. Castleman and K. H. Bowen, J. Phys. Chem. 100, 12911 (1996).

${ }^{2}$ M. D. Morse, M. E. Geusic, J. R. Heath, and R. E. Smalley, J. Chem. Phys. 83, 2293 (1985).

${ }^{3}$ W. A. de Heer, Rev. Mod. Phys. 65, 611 (1993).

${ }^{4}$ A. Herzing, C. J. Kiely, A. F. Carley, P. Landon, and G. J. Hutchings, Science 321, 1331 (2008).

${ }^{5}$ S. A. Claridge, A. W. Castleman, Jr., S. N. Khanna, C. B. Murray, A. Sen, and P. S. Weiss, ACS Nano 3, 244-255 (2009).

${ }^{6}$ H. Y. Yang, Y. Wang, H. Q. Huang, L. Gell, L. Lehtovaara, S. Malola, H. Häkkinen, and N. F. Zheng, Nat. Commun. 4, 2422 (2013), and references therein.

${ }^{7}$ P. Jensen, Rev. Mod. Phys. 71, 1695 (1999).
${ }^{8}$ V. N. Popok, I. Barke, E. E. B. Campbell, and K.-H. Meiwes-Broer, Surf. Sci. Rep. 66, 347 (2011).

${ }^{9}$ P. Fayet, F. Granzer, G. Hegenbart, E. Moisar, B. Pischel, and L. Wöste, Phys. Rev. Lett. 55, 3002 (1985).

${ }^{10}$ W. Eberhardt, P. Fayet, D. M. Cox, Z. Fu, A. Kaldor, R. Sherwood, and D. Sondericker, Phys. Rev. Lett. 64, 780 (1990).

${ }^{11}$ J. T. Lau, A. Föhlisch, R. Nietubyc, and W. Wurth, Phys. Rev. Lett. 89, 057201 (2002).

${ }^{12}$ S. Pratontep, P. Preece, C. Xirouchaki, R. E. Palmer, C. F. Sanz-Navarro, S. D. Kenny, and R. Smith, Phys. Rev. Lett. 90, 055503 (2003).

${ }^{13}$ U. Busolt, E. Cottancin, H. Röhr, L. Socaciu, T. Leisner, and L. Wöste, Appl. Phys. B 68, 453 (1999).

${ }^{14}$ R. Schaub, H. Jödicke, F. Brunet, R. Monot, J. Buttet, and W. Harbich, Phys. Rev. Lett. 86, 3590 (2001).

${ }^{15}$ B. Yoon, H. Häkkinen, U. Landman, A. S. Wörz, J-M. Antonietti, S. Abbet, K. Judai, and U. Heiz, Science 307, 403 (2005).

${ }^{16}$ W. E. Kaden, T. Wu, W. A. Kunkel, and S. L. Anderson, Science 326, 826 (2009).

${ }^{17}$ X. Tong, L. Benz, P. Kemper, H. Metiu, M. T. Bowers, and S. K. Buratto, J. Am. Chem. Soc. 127, 13516 (2005).

${ }^{18}$ W. Yamaguchi and J. Murakami, J. Am. Chem. Soc. 129, 6102 (2007).

${ }^{19}$ Y. Yang, J. Zhou, M. Nakayama, L. Nie, P. Liu, and M. G. White, J. Phys. Chem. C 118, 13697-13706 (2014).

${ }^{20}$ A. Lando, N. Kebaili, Ph. Cahuzac, A. Masson, and C. Brechignac, Phys. Rev. Lett. 97, 133402 (2006).

${ }^{21}$ R. Reichel, J. G. Partridge, F. Natali, T. Matthewson, S. A. Brown, A. Lassesson, D. M. A. Mackenzie, A. I. Ayesh, K. C. Tee, A. Awasthi, and S. C. Hendy, Appl. Phys. Lett. 89, 213105 (2006).

${ }^{22}$ X. Tang, X. Li, Y. Wang, K. Wepasnick, A. Lim, D. H. Fairbrother, K. H. Bowen, T. Mangler, S. Noessner, C. Wolke, M. Grossmann, A. Koop, G. Gantefoer, B. Kiran, and A. K. Kandalam, J. Phys.: Conf. Ser. 438, 012005 (2013).

${ }^{23}$ T. Darby and C. Wayman, J. Cryst. Growth 29, 98 (1975).

${ }^{24}$ L. Bardotti, B. Prevel, M. Treilleux, P. Melinon, and A. Perez, Appl. Surf. Sci. 164, 52 (2000).

${ }^{25}$ M. F. Juarez, S. Fuentes, G. J. Soldano, L. Avalle, and E. Santos, Faraday Discuss. 172, 327 (2014).

${ }^{26}$ M. Schmidt, R. Kusche, B. v. Issendorff, and H. Haberland, Nature 393, 238 (1998).

${ }^{27}$ A. Aguado and M. F. Jarrold, Annu. Rev. Phys. Chem. 62, 151 (2011).

${ }^{28} \mathrm{X}$. Li, K. Wepasnick, X. Tang, D. H. Fairbrother, K. H. Bowen, A. Dollinger, C. H. Strobel, J. Huber, T. Mangler, Y. Luo, S. Proch, and G. Gantefoer, J. Appl. Phys. 115, 104304 (2014).

${ }^{29}$ K. A. Wepasnick, X. Li, T. Mangler, S. Noessner, C. Wolke, M. Grossmann, G. Gantefoer, D. H. Fairbrother, and K. H. Bowen, J. Phys. Chem. C 115, 12299 (2011)

${ }^{30}$ K. Boggavarapu, A. Kandalam, R. Rallabandi, P. Koirala, X. Li, X. Tang, Y. Wang, D. H. Fairbrother, G. Gantefoer, and K. Bowen, J. Chem. Phys. 136, 024317 (2012)

${ }^{31}$ X. Tang, J. Schneider, A. Dollinger, Y. Luo, A. S. Wörz, K. Judai, S. Abbet, Y. D. Kim, G. F. Ganteför, D. H. Fairbrother, U. Heiz, K. H. Bowen, and S. Proch, Phys. Chem. Chem. Phys. 16(14), 6735-6742 (2014). 